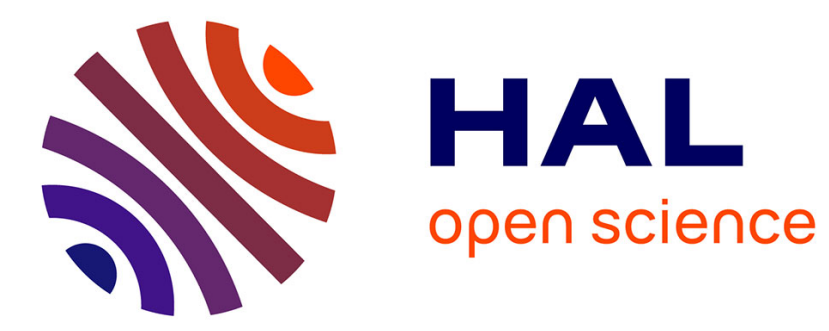

\title{
Crypto assets: the role of ICO tokens within a well-diversified portfolio
}

Saman Adhami, Dominique Guegan

\section{To cite this version:}

Saman Adhami, Dominique Guegan. Crypto assets: the role of ICO tokens within a well-diversified portfolio. 2019. halshs-02353656

\section{HAL Id: halshs-02353656 \\ https://shs.hal.science/halshs-02353656}

Submitted on 7 Nov 2019

HAL is a multi-disciplinary open access archive for the deposit and dissemination of scientific research documents, whether they are published or not. The documents may come from teaching and research institutions in France or abroad, or from public or private research centers.
L'archive ouverte pluridisciplinaire HAL, est destinée au dépôt et à la diffusion de documents scientifiques de niveau recherche, publiés ou non, émanant des établissements d'enseignement et de recherche français ou étrangers, des laboratoires publics ou privés. 


\section{Documents de Travail du

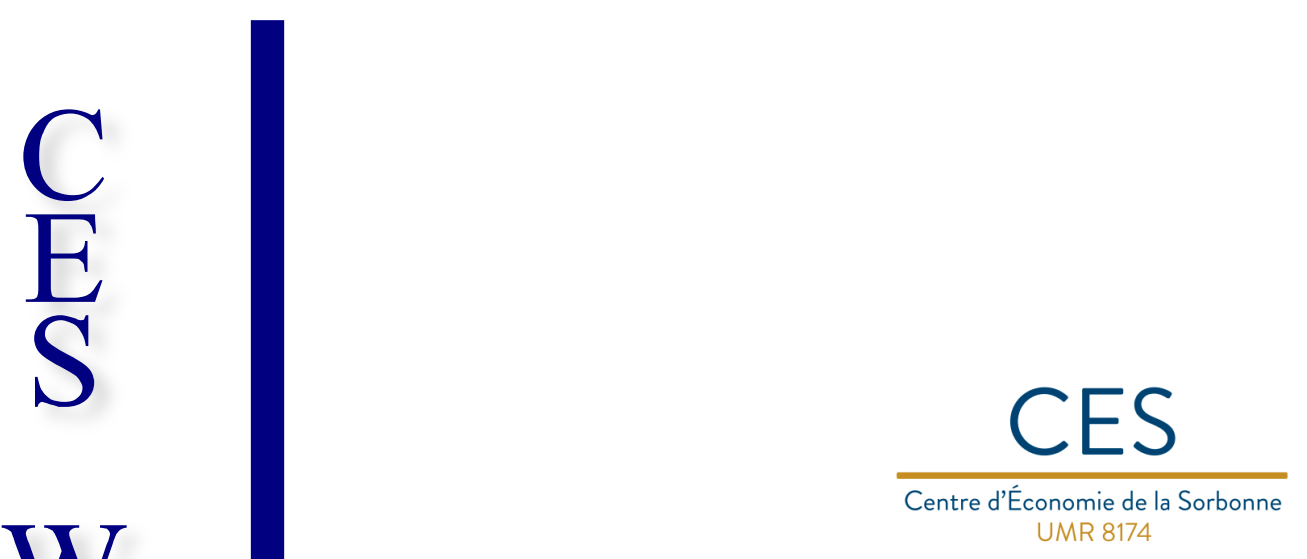

Crypto assets: the role of ICO tokens within a well-diversified portfolio

Saman ADHAMI, Dominique GUEGAN

2019.20

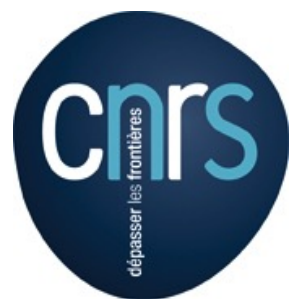




\title{
Crypto assets: the role of ICO tokens within a well-diversified portfolio
}

\author{
Saman Adhami* and Dominique Guegan** \\ * Vienna Graduate School of Finance - corresponding author \\ saman.adhami@vgsf.ac.at \\ ** Université Paris1 Panthéon-Sorbonne, France \\ Associate Professor Ca'Foscari Unversity of Venezia, Italy
}

Associate Researcher University of Economics Ho Chi Minh City, Vietnam

dominique.guegan@univ-paris1.fr

Keywords: Cryptocurrency, Initial Coin Offering, DCC-MGARCH, Safe Haven, Hedge

JEL Codes: G11, G15

On behalf of all authors, the corresponding author states that there is no conflict of interest.

\begin{abstract}
This paper re-examines the discussion on blockchain technology, crypto assets and ICOs, providing also evidence that in crypto markets there are currently two classes of assets, namely standalone cryptocurrencies (or 'coins') and tokens, which result from an ICO and are intrinsically linked to the performance of the issuing company or venture. While the former have been arguments of various empirical studies regarding their price dynamics and their effect on the variance of a well-diversified portfolio, no such study has been done to analyze listed tokens, which in our sample are over 700 and with a backing of about $\$ 17.3 \mathrm{Bn}$ from their respective ICOs. Therefore, investors interested in optimizing their portfolios should first assess the diversifier, hedge or safe haven role of tokens vis-àvis traditional assets, on top of 'coins', in order to sensibly use this new asset class. After constructing various indices to represent both the token asset class as a whole and its sub-classes, we model dynamic conditional correlations among all the assets in our sample to obtain time-varying correlations for each token-asset pair. We find that tokens are effective diversifiers but not a hedge or a safe haven asset. We evidence that tokens retain important systematic differences with the two other asset classes to which they are most generally compared to, namely 'coins' and equities.
\end{abstract}

\section{Introduction}

Distributed ledger technologies (DLT), to which blockchain is a major example, are still a novelty within the financial world. Different experimentations by developers and entrepreneurs since 2010 allowed many of their ideas to take the form of crypto-assets, thus generating financial innovation. 
Consequently new platforms have been created for trading, storage, betting, or simply making some bureaucratic process or information indelible and transparent, all relying on DLT.

Among the many outlets for this technology, Initial Coin Offerings (ICO) got prominence as they allowed to securely code a pseudo-security agreement between entrepreneurs and external contributors of funds. All of this happens within a cryptographic 'token', which then is usually traded online for speculative purposes other than being exchanged for the underlying goods or services promised by the issuer. Since 2015 , small and medium-sized enterprises found that they could finance their technologically-intensive operations through the ICO funding mechanism, which until the first half of 2017 appeared to be an extraordinarily easy market to tap for funds, mainly due to the crypto-frenzy of the period and lack of clear regulatory guidelines. As of the $15^{\text {th }}$ of September 2019 , \$31.5Bn have been collected through ICOs from over 1770 funding rounds ${ }^{1}$.

ICOs are a novel funding mechanism used by technology-savvy entrepreneurs to finance projects that are mainly revolving around the blockchain technology. Before an ICO, an ad-hoc crypto asset called 'token' is created on a distributed ledger, and all its uses and transfer options are encoded in it from the onset. Contributors send 'mainstream' crypto-assets, such as Bitcoin and Ether among others, or even fiat currencies, to the e-wallet address of the company and automatically received a predetermined amount of tokens in exchange. Each ICO-ed token has its own underlying characteristics and will give rights to third parties that are specific for each project: some are created to grant rights akin to a currency, others resembling equity instruments, and others are more similar to a utility asset, which allows its holder to have access to certain non-financial advantages, including access to a platform, right to vote, right to obtain value-add information. According to Li and Mann (2018), selling rights to a digital service before having an operational platform can be an efficient mechanism. Although there exist detailed descriptions of the different classes of rights attached to tokens (see Adhami et al. 2018, and Giudici and Adhami 2019), market practitioners generally only recognize three types of tokens, namely utility, security and payment. Therefore, while seeking financial returns through capital gains, the average crypto investor categorizes tokens as either granting rights to a valuable service (utility token), granting equity-like ownership stake in the entrepreneurial project (security token), or granting a medium of value exchange (payment token).

An example of ICO project issuing a currently listed token is SureRemit, a project born in California in 2017 aiming at creating a digital ecosystem for non-cash remittances. The token is built on the Stellar DLT, whose XLM coin was released in July 2014, making the SureRemit ICO one of the few not using the ETH blockchain as support. This decision was justified in the related whitepaper (or offering memorandum) based on the sophistication of the Stellar network, its use by reputable companies such as IBM and Deloitte, and especially the lower transaction costs of transfers completed on it, which becomes crucial for the relatively small size of remittance transactions. The SureRemit token (RMT) is an example of pure utility token: its only use is within the platform between remittance senders, receivers and merchants using the XLM who have partnered with SureRemit. If the market deems the platform of the venture worthwhile and predicts demand increase for its service, the demand for RMTs will surge and those holding the tokens (either because they bought them during the ICO or in the secondary market) will be able to sell their excess supply at a capital gain. In this sense, according to the Howey test of the US securities law, the purchase of tokenized assets with the expectation of obtaining financial returns may be enough to deem the tokens securities. Nevertheless, these tokens are technically not rights to the profits of the company, nor a financial obligation for the company, nor do they provide voting rights within the company's decision-making bodies. The ICO of the company was concluded in just 6 days on the $16^{\text {th }}$ of June 2018, raised

\footnotetext{
${ }^{1}$ Data from www.coinschedule.com.
} 
between 7 to 10 million USD (depending on the conversion rate of the collected cryptocurrencies) with a token retention rate of $50 \%$ for the venture treasury and insiders. The RMT was listed on a crypto exchange on the $15^{\text {th }}$ of February 2018 with a first-day underpricing of 55\%, but the price and market capitalization fell from $\$ 18$ million at the onset to just $\$ 0.9$ million by the end of July 2019 . Overall, despite being of just 2 years of age at the time of this writing, the startup managed to raise VC-size funding without any formal financial intermediary, have its asset listed and traded on a public exchange and, despite the fall of its token price, it is still operational and expanding its network of partnered merchants.

After several studies on the legal issues related to the crypto-asset issuance, one of the focal concerns that will have to be handled by legal, economic and financial experts in the coming years is the understanding of the various outcomes of ICOs, of tokenization and, more globally, of distributed ledger technology on the governance of companies. This changes in the governance structure are key characteristics of this new way for raising funds because they rely on an algorithmic governance. The governance structure of blockchain companies makes the investigation of the evolution of the tokens as important as the ICO fundraising event itself. Indeed, recall that the main goal in corporate governance is to support economic efficiency, sustainable growth and financial stability. The discussion around the regulation for ICOs, and then of the traded tokens is in progress across many jurisdictions, see for instance Atzori (2016), Buterin (2017), Blemus and Guégan (2019), among others.

In the literature, most of the studies regarding tokens concern the success of fundraising for ICO events, led by Adhami et al. (2018) and Fisch (2018). There are also studies looking at the motivation of potential subscribers when deciding to 'invest' in tokens, see for instance Fenu et al. (2018), Yadav (2017), among others. As the ICO mechanism is often considered similar to the crowdfunding process (even if it is not entirely correct), some studies have been initiated to determine the relationship between entrepreneurs and the many dispersed investors, with heterogeneous objectives but all related to the general characteristics or governance structure of the newly formed blockchain ventures, e.g. Blemus and Guégan (2019), Catalini and Gans, (2018), Giudici and Adhami (2019) and Howell et al. (2018). Ex-post revelation that even some successfully funded ICOs were indeed scams or frauds is the major determinant of publicly traded tokens being delisted. In terms of delisting determinants, a major role is played by the idiosyncratic risk of entrepreneurial ventures, which makes, on average, $90 \%$ of the start-ups fail. The huge amount of funds received through an ICO can surely delay legitimate bankruptcies, but not eliminate the possibility. Moreover, many tokens are listed months after a successful public offering and the listing requirements of crypto exchanges are much more lax than traditional exchanges. All of these contribute to a very volatile secondary market for tokens.

The investigation of the evolution of the token prices post-fundraising is a crucial task for the corroboration of these new start-ups, and also to understand how a new form of governance can emerge for these companies, as we know that stock market discipline has been an important governance lever for traditional listed companies (e.g. Shleifer and Vishny 1997). This new market is interesting because in its essence it is totally different than the cryptocurrencies supported by mining like Bitcoin, Ethereum, Ripple, etc. for which predictability is questionable. Tokens being created and exchanged for cryptocurrencies and fiat currencies have a different status, as their price is in a large part intrinsically bound to the underlying entrepreneurial project of the issuing company. Tokens, as a new crypto asset, create the strongest link between the industrial world and blockchain-led innovation, both in terms of financing and monetary policy. Thus, studying the evolution of their prices provides rich information on the wealth and dynamics of this market, and how it compares with other existing assets. 
There has been a lot of empirical work on Bitcoin and other coins with regards to their role in welldiversified portfolio (e.g. Bouri et al. 2017, Wong et al. 2018, and Urquhart and Zhang 2019), i.e. their interaction with traditional asset classes. Nevertheless, at this time, despite investors on a global scale contribute billions to ICOs and trade tokens $24 / 7$ on crypto exchanges, there exists no specific study that explores tokens as the other major crypto asset class that they effectively are. This is the subject of this paper, i.e. to empirically uncover some of the most important properties of the token market from the point of view of investors that are mean-variance optimizers. In order to investigate the influence of the tokens on the stability of the crypto-assets world, in this paper we propose the creation of sensible indices based on the universe of listed tokens and then to analyse their dynamic behaviour alongside full-fledged cryptocurrencies ('coins') and other traditional assets. Throughout the paper we consider a diversifier to be an asset that is positively but not perfectly correlated with another asset, a hedge to be an asset that is uncorrelated or negatively correlated with another asset, and a safe haven to be an asset that is uncorrelated or negatively correlated with another asset in times of market stress or turmoil (Baur and Lucey, 2010).

The paper is organized as follows: in Section 2 we explain how the data was obtained; Section 3 present our methodology of index creation and the modelling of dynamic conditional correlations between all the assets in our sample; the analyses in Section 4 provide our main results, while Section 5 is devoted to the conclusion.

\section{Data}

Throughout the paper we emphasize that there are currently two categories of crypto assets on the market, namely 'coins' and 'tokens'. While coins always rely on their own, ad-hoc built blockchain and can be thus used as full-fledged cryptocurrencies, tokens are bundles of rights embedded in a cryptographic asset that is intrinsically linked to the success of an entrepreneurial project. Moreover, frequently the technological platform from which tokens are issued and on which they are later used (e.g. in exchange for services by the issuing company) relies on a pre-existing blockchain operating through a pre-existing coin.

The major data provider on crypto asset prices is the website CoinMarketCap.com, which aggregates the price of each coin or token across all the different cryptocurrency exchanges listing the asset. Both practitioners and academics heavily rely on this website to obtain the time series of crypto assets, and so did we. Information regarding major ICO-ed tokens is readily available, but many tokens who underwent a successful ICO did not get listed and many of the listed tokens were eventually delisted. Delisting occurs after a prolonged steep drop of the token price or when the project is labelled as a scam or fraud by influential market observers. Idiosyncratic bankruptcy of the backing company or failure of the entrepreneurial project can also lead to tokens being delisted from major cryptocurrency exchanges. Moreover, since listing does not always occur immediately after the end of the fundraising event (as it would for IPOs), reconstructing the price history of tokens become even more challenging. In order to have the complete census of listed tokens, we extracted all the assets listed at any time on CoinMarketCap.com (as the website does not properly distinguish between ICO-ed tokens and general coins), and then identified those crypto assets originating from an ICO using ICOBench.com as validation tool. Only for this sub-group, the price time series were extracted from CoinMarketCap.com. The panel of time series obtained is unbalanced, as each token has its own entry (and exit) point in the traded pool. Overall, we identified 776 tokens that were at any time listed ${ }^{2}$, with the time series starting on February $18^{\text {th }} 2015$ with a single token and ending on February $27^{\text {th }} 2019$ with 693 (listed) tokens. Out of these tokens, complete data regarding market

\footnotetext{
${ }^{2}$ We exclude Ethereum (ETH) as we consider it a 'coin' rather than a 'token'.
} 
capitalization and traded volume were available for 700 assets. Furthermore, information regarding the characteristics of the sampled tokens and their respective ICOs were obtained from ICOBench.com.

Data regarding major coins were obtained through CoinMarketCap.com through a much simpler algorithm. We used 11 coins as representatives of the cryptocurrency market relying mostly on their market capitalization and the frequency of their inclusion in the CRIX index ${ }^{3}$. These are: Bitcoin, Bytecoin, Dash, Dogecoin, Ethereum, Litecoin, MaidSafeCoin, Monero, Ripple, Stellar, and ZCash.

Figure 1 depicts how the ICO market, as represented by fundraising volumes, historically moved with the price of Bitcoin. Surges in Bitcoin prices are mimicked by contemporaneous increased ICO activity, as they both indicate strong market interest in the crypto world and stronger purchasing power of cryptocurrencies used in ICO contributions. Throughout the history of ICOs, cryptocurrencies and Bitcoin were highly volatile, but this did not induce important volume effects on ICO activity.

-Figure 1 about here-

Moreover, as Figure 2 shows, even in the quarters with highest ICO funding volumes and highest numbers of ICOs completed, the response in terms of token listings is moderate. This suggests that the token listing decision, although inevitable given the intrinsic characteristics of tokens, is not immediate and requires market timing and other strategic considerations by the entrepreneurial team.

-Figure 2 about here-

Time series for the traditional asset classes were obtained from Bloomberg. In terms of equity and bond indices, we distinguished between global and regional portfolios. To represent global equities we used the S\&P Global 1200 while for European and US equities we used, respectively, the $S \& P$ Europe 350 and the S\&P 500. To represent global bonds we used the S\&P Intl. Corporate Bond index, while for Europe and the US we used the S\&P Eurozone Investment Grade Corporate Bond Index and the $S \& P$ Investment Grade Corporate Bond Index. To represent real estate assets, gold and the commodity markets we used the S\&P Global REIT, the S\&P/TSX Global Gold Index and the S\&P GSCI (in the case of Europe and the US the real estate market is proxied by the S\&P Developed Property). Alternative index specifications for the different asset classes were used in the robustness checks.

\section{Methodology}

\subsection{Index construction}

In order to study the properties of the token market, 4 token indices were created out of the 700 sampled ICO-backed assets. The first index contains all 700 tokens, while the remaining three are formed as k-means clusters of the former group according to the coefficients of variation of daily market capitalization and trading volume. As the crypto market is generally highly volatile, a clustering based on mere average market capitalization did not seem the best option, and the addition of the volume dimension appeared to enrich the grouping. Eventually, coefficients of variation incorporate also the dispersion around volume and market cap for each token, and thus help generate clusters of tokens that have more similar distributions. To further check that the grouping algorithm does not influence our results, alternative definitions of the three sub-group token indices were also used. For instance, another set of three indices were obtained by minimizing

\footnotetext{
${ }^{3}$ We excluded CRIX components that are effectively tokens (e.g. BAT, NEO and QTUM). For more information on the CRIX indices see Trimborn and Härdle (2018).
} 
within-cluster standard deviation of the Gini coefficient of the average daily market capitalization of each token series ${ }^{4}$. For any clustering algorithm, 3 clusters or groups resulted to be optimal, and in our main specification these would be the tokens with low market cap, volume and related stability (521 constituents), tokens with medium market cap, volume and related stability (151 constituents) and tokens with high market cap, volume and related stability of these values (28 constituents). This subdivision of indices that mimics small, mid and large-cap distinctions is necessary to understand more specifically how different types of tokens behave and if one particular cluster of tokens would impact dynamic correlations with traditional assets and coins differently, perhaps attenuating or reinforcing the diversification power of the total token index.

Daily returns for each token were computed as follows ${ }^{5}$ :

$$
r_{i, t}=\frac{P_{i, t}}{P_{i,(t-1)}}-1
$$

where $P_{i, t}$ is the price of the token at time t. During the sampling period the only major event that could happen for a token is delisting, therefore there is no need to adjust for dividends or splits. Once a token is delisted, its return will simply drop to $-100 \%$. Afterwards, for each trading day, in a specific group, the single returns are aggregated as a value-weighted return, $R_{t}$, according to:

$$
R_{t}=\sum_{i=1}^{N_{t-1}} W_{i, t-1} r_{i, t}
$$

where $W_{i, t-1}$ is the dynamical cross-sectional market cap weight of the $i^{\text {th }}$ token on the previous trading day, and $N_{\mathrm{t}-1}$ is the number of assets within the group of reference. This dynamical approach, while being more realistic, it will also allow the investor to adjust the evolution of the portfolio with respect of the behaviour of other assets. Finally, the index $\left(I_{t}\right)$ for the return price series of each group is obtained as:

$$
I_{t}=1000 *\left(1+R_{1}\right)\left(1+R_{2}\right) \ldots\left(1+R_{t}\right)
$$

The same methodology is used to obtain an index representing coins from the 11 cryptocurrencies previously mentioned. Finally, given the price series of the indices, logarithmic return series are obtained.

\subsection{The dynamic conditional correlation models}

After modelling the return and volatility of the individual assets, we employ the dynamic conditional correlation (DCC) model of Engle (2002) and the asymmetric dynamic conditional correlation (ADCC) model of Cappiello, Engle and Sheppard (2006) to derive the conditional correlations between the token indices and each of the other assets. DCC multivariate GARCH models have recently become popular tools to study the co-movements between multiple assets, especially thanks to their flexibility, which is comparable to univariate GARCH models (Engle, 2002), and the fact that they do not generally introduce computational complications (Parhizgari and Cho, 2008), or unreasonable estimates as models like BEKK and CCC may do. A number of recent empirical papers used the DCC

\footnotetext{
${ }^{4}$ The change in index composition and influence on the time series behaviour of the series was negligible.

${ }^{5}$ Simple or arithmetic returns are computed in this step as the index creation procedure requires crosssectional aggregation of constituent asset returns for each date.
} 
methodology to explore the volatility dynamics of Bitcoin and other coins, e.g. Bouri et al. (2017) or Urquhart and Zhang (2019).

Specifically, given a $n \times 1$ vector of returns, $r_{t}$, its AR(1) process can be written as:

$$
r_{t}=\mu+a r_{t-1}+\varepsilon_{t}
$$

We then model residuals in terms of the conditional covariance matrix of $r_{t}\left(H_{t}\right)$ and a random vector of error $\left(z_{t}\right)$ as follows:

$$
\varepsilon_{t}=H_{t}^{1 / 2} z_{t}
$$

The DCC model requires, firstly, the estimation of the GARCH parameters and then the estimation of the conditional correlations using the following relations:

$$
\begin{gathered}
H_{t}=D_{t} R_{t} D_{t} \\
D_{t}=\operatorname{diag}\left(h_{1, t}^{1 / 2}, \ldots, h_{n, t}^{1 / 2}\right) \\
R_{t}=\operatorname{diag}\left(q_{1, t}^{-1 / 2}, \ldots, q_{n, t}^{-1 / 2}\right) Q_{t} \operatorname{diag}\left(q_{1, t}^{-1 / 2}, \ldots, q_{n, t}^{-1 / 2}\right)
\end{gathered}
$$

Where $R_{t}$ is the conditional correlation matrix, $D_{t}$ is the diagonal matrix with time-varying standard deviation on the diagonal, and the expressions of $h$ are univariate GARCH models, which in the case of $\operatorname{GARCH}(1,1)$ can be written as:

$$
h_{t}=\omega+\alpha_{i} \varepsilon_{i, t-1}^{2}+\beta_{i} h_{i, t-1}^{2}
$$

Moreover, $Q_{t}$ is a symmetric positive definite matrix such that:

$$
Q_{t}=\left(1-\theta_{1}-\theta_{2}\right) \bar{Q}+\theta_{1} z_{t-1} z_{t-1}^{\prime}+\theta_{2} Q_{t-1}
$$

where $\bar{Q}$ is an $n \times n$ unconditional correlation matrix of the standardized residuals $z_{i, t}$ with

$$
z_{i, t}=\varepsilon_{i, t} / \sqrt{h_{i, t}}
$$

In addition, $\theta_{1}$ and $\theta_{2}$ are non-negative parameters determining the smoothing process used to construct the dynamic conditional correlations. As long as $\theta_{1}+\theta_{2}<1$, the DCC model is meanreverting. Finally, the correlation estimator results to be:

$$
\rho_{i, j, t}=\frac{q_{i, j, t}}{\sqrt{q_{i, i, t}+q_{j, j, t}}}
$$

The model extension of Cappiello et al. (2006) relies on the insertion of a term capturing asymmetric responses that results in:

$$
h_{t}=\omega+\alpha_{i} \varepsilon_{i, t-1}^{2}+\beta_{i} h_{i, t-1}^{2}+k_{i} \varepsilon_{i, t-1}^{2} I\left(\varepsilon_{i, t-1}\right)
$$

where the indicator function $I\left(\varepsilon_{i, t-1}\right)$ is equal to 1 if there is a negative shock and zero otherwise, and a positive value for $k$ would determine that negative residuals tend to increase variance more than positive ones. The asymmetric effect is necessary to capture a frequently present phenomenon for financial assets, namely that unexpected drops in asset prices tend to increase volatility more than unexpected increases in prices of the same magnitude. The previously defined matrix $Q_{t}$ changes in the following way for the ADCC model: 


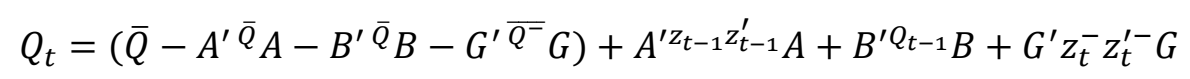

where $A, B$ and $G$ are $n \times n$ parameter matrices, $z_{t}^{-}$are zero-threshold standardized errors (which are equal to $z_{t}$ when less than zero and zero otherwise), and $\bar{Q}$ and $\overline{Q^{-}}$are the unconditional matrices of $z_{t}$ and $z_{t}^{-}$.

Eventually, both DCC and ADCC models allow us to obtain time series of conditional correlations for each pair of assets within the sample.

\subsection{Hedge and safe haven detection model}

In order to thoroughly assess the hedging and safe haven characteristics of token returns we follow the procedure used by Baur and McDermott (2010) to assess the features of gold as an asset class ${ }^{6}$. Nevertheless, our algorithm resembles more closely that of Ratner and Chiu (2013) who examined the hedging properties of CDS versus the stock market using DCC as estimation procedure for the comovements of assets.

In practice, for each asset class, we regress its dynamic conditional correlation with the token index on a constant and three dummy variables indicating extreme circumstances of the reference asset. The specification is as follows:

$$
\rho_{t}=m_{0}+m_{1} D\left(r_{\text {asset }} p_{10}\right)+m_{2} D\left(r_{\text {asset }} p_{5}\right)+m_{3} D\left(r_{\text {asset }} p_{1}\right)
$$

In this specification the $\mathrm{D}($.) is a binary variable equal to one in any period in which the underlying asset has experienced extreme negative movements (i.e. returns lower or equal to the $10^{\text {th }}, 5^{\text {th }}$ and $1^{\text {st }}$ percentile of the return distribution). The token index would act as a weak hedge if $m_{0}$ is zero and a strong hedge in case $m_{0}$ results in a significant negative value ${ }^{7}$. Moreover, the token asset class would prove to be a weak safe haven if the coefficients $m_{1}, m_{2}$, or $m_{3}$ result in non-positive values, or a strong safe haven in case they are negative and significant. In case these three coefficients result in positive values, they would evidence that tokens are not a safe haven asset vis-à-vis the specific reference asset. Finally, if either of the coefficients of the quantile dummies is significantly different from zero, it would indicate that tokens and the market of the asset of reference have a non-linear relationship.

\section{Empirical Results}

\subsection{Descriptive statistics}

Our main clustering methodology results, as anticipate earlier, to a total index of 700 constituents, and low, medium and high indices of 521, 151 and 28 tokens respectively.

Table 1 presents some basic information regarding the sampled listed tokens, mostly in terms of the ICO event that generated them and the entrepreneurial capital behind them. For the four indices, the main geographical localisation is Europe with 32.2\% (low index) and 38.9\% (Mid index), then North America and Singapore. A large proportion of the large market cap and high stability tokens (high index) comes from Europe and the ICO-friendly micro-states. Huang et al. (2019) confirm that the emergence and frequency of ICOs is intrinsically related to geography, or more precisely to institutional or regulatory framework of a country. The total amount raised by the sampled tokens during their ICOs is $\$ 12.7$ billion, with a mean of $\$ 25.6$ million per venture. Another interesting

\footnotetext{
${ }^{6}$ Baur and McDermott (2010) do not use dynamic conditional correlations but rather time varying betas calculated from rolling regressions.

${ }^{7}$ Additionally, the sum of parameters $m_{1}$ to $m_{3}$ must not be jointly positive and exceeding the value of $m_{0}$ in order for the tokens to be a hedge.
} 
feature of the listed tokens is that about $89 \%$ of them rely on the Ethereum blockchain, and therefore on its platform and coin. On the other hand, as stressed earlier, coins are always standalone in terms of their DLT setup. Moreover, most of the tokens that made it from the ICO to listing on crypto exchanges are utility tokens (95.7\%), i.e. their main purpose is to serve as tools for operating or using the service platform generated by the issuing company. Finally, as a further note on the consistency of our clustering, the average experts rating ${ }^{8}$ of the tokens is lowest for the low market cap and stability tokens, improves for medium tokens and becomes the highest for the tokens of the high index, which as we commented earlier are the largest and most stable.

- Table 1 about here-

Moreover, a standard routine of univariate analyses were done for each index and each of the other time series used, including stationarity tests and modelling the most appropriate individual ARIMAGARCH. Nevertheless, the focus of the paper is not on forecasting, but rather on co-movements of returns, so these intermediate results were not reported.

Figure 1 provides the evolution of the prices of the four token indices. The range of values evolve between 0 and 5000. For all there is a peak in January 2018, but this one is amplified for the medium tokens index. There seem to be some seasonality between mid-2017 until the beginning of 2019, which corresponds to important volatility events in the prices. The time series behaviour of the low token index mimics almost perfectly the dynamics of the total token index, suggesting what is later confirmed with the re-estimation of the DCC model with each sub-index as counterparty, i.e. that small and volatile tokens determine the behaviour of the whole token market and most of the comovement with traditional assets and coins.

- Figure 3 about here-

Figure 2 adds the evolution of the prices of Bitcoin in purple and the value-weighted index of coins built using the 11 coins mentioned in the data section. The scale of the range has increased exponentially and thus the comparison with the previous figure does not permit now to detect correctly the evolution of the tokens index. Nevertheless the same pattern is observed on the two graphs with the peak around the beginning of 2018. It is clear that coins and tokens have different mean and volatility dynamics.

- Figure 4 about here-

In Figure 3 we present the returns for the full token index (700 tokens), the cryptocurrency index (11 cryptocurrencies) and the Bitcoin. Bitcoin (green line) seems the least volatile. However, tokens (black line) present an important volatility between 2015 up to mid-2017, and then it is the coin index which seems most volatile (red line). Therefore, the behaviour of these three indices seems quite different and the latter two indices do not follow the evolution of Bitcoin. Since value-weighted indices are generally dominated by stocks or assets with the largest market capitalization, the Bitcoin clearly dominates the coin index in terms of composition. However, the weight of the Bitcoin within this index dropped from 85\% in February 2015 to 66\% in February 2019, explaining why the divergence between the Bitcoin series and the coin index becomes relevant after the first half of 2017.

- Figure 5 about here-

Table 2 provides some descriptive statistics regarding the distributions of the daily return time series used in the analysis. Looking at the skewness, none of these indices are symmetrical, most of them

\footnotetext{
${ }^{8}$ Ratings from ICOBench.com.
} 
are left skewed, except the mid token index and US bonds. Looking at the kurtosis, except for the high token index, all the other indices have a kurtosis bigger than 3 with highest values for the total token index and the low token index. So these first remarks underline that the Gaussian distribution cannot characterize most of the tokens, nor a symmetrical distribution. Perhaps a better fit would come from an asymmetrical NIG distribution which can be fitted to data with different skewness and high kurtosis ${ }^{9}$. Now looking at the ADF test, we can assume stationarity for the daily returns, and the estimated parameter of the $A R(1)$ process (which permits to provide a basic information on the evolution of the level of the value at the next day) present negative values for the token indices, the coin index, two equities, one bond, the gold and the commodities. The values of the parameters are $10 \%$ higher for the total and the low token indices relative to the others. The Ljung-box test at lag 12 indicates that for the mid and high indices, global and US bonds, and commodities, there is some residual informational value in the autoregressive lags. The table evidences that so far, since being listed, tokens had on average negative returns, which is reasonable given the gradual drop of the crypto frenzy phenomenon and the fact that these tokens represent entrepreneurial endeavours at a very young company age.

- Table 2 about here-

\subsection{Dynamic conditional correlation}

As indicated by the descriptive statistics, alternative ARMA specifications for the underlying process result is negligible differences in the estimation of the GARCH and DCC parameters. We proceed by fitting either the same $\operatorname{ARMA}(1,1)$ for all the time series or to adapt the each ARMA specification to the specific series, and compare these with different multivariate distributions and GARCH models.

In Table 3 we provide the results of the best fitting models for the different DCC specifications combined with related GARCH, and also for the ADCC models with some related GARCH modelling. These models all have multivariate Student's t distributions as reference. In particular, the GJRGARCH allows to account for the asymmetry in returns, while the EGARCH allows to capture asymmetries in volatility clustering. Looking at the AIC and BIC criteria, we retain the ADCC-ARMA(1,1)-GJRGARCH specification ${ }^{10}$. This means that the returns are modelled with an $\operatorname{ARMA}(1,1)$, the volatility with a GJRGARCH $(1,1)$ modelling which is interesting in our case because it permits to take into account the influence of the positive and negative shocks in an asymmetric way, which correspond to the drops that we have observed on the Figures 1, 2 and 3 . The conditional correlation is obtained through the expression provided for the matrix $Q_{t}$ in Section 3.

- Table 3 about here-

We do not elaborate on the estimated coefficients of the DCC model as our interest relies on the time series of the pairwise correlations with the constructed token series. In Table 4 we provide statistics on the daily conditional correlations between each asset and the total token index.

-Table 4 about here-

Panel A presents the results of the DCC for a globally diversified investor. The conditional correlations between tokens and other assets range from 0.532 (vs. coins) to 0.067 (vs. real estate assets). For equities, bonds, gold and commodities, the value in mean is around 0.1. This means that there is nearly no correlation between the total token index and all the assets except with coin. In this table

\footnotetext{
${ }^{9}$ For a detailed description of the normal inverse Gaussian (NIG) distribution see Folks and Chhikara (1978) and Shuster (1968).

${ }^{10}$ Other specification, such as a NIG underlying distribution, were tested, but only the above class of models is presented here due to its superiority in terms of information criteria.
} 
we exhibit also the repartition of the correlation looking at different quantiles. For the coin-token correlation we see a high value at the Q75 (0.807) which attains 0.902 in the right tail. Concerning Panel $B$, which contains the Eurozone assets, the repartition is nearly the same. We have a mean value of 0.513 for the correlation between the total token index and the coins and a value of 0.08 in mean for the equities, the bonds, real estate, gold and the commodities. Again, except for coins, there is even less correlation (nearly none) between tokens and traditional assets. With regards to Panel $\mathrm{C}$, which takes the point of view of domestically-biased US investor, the behaviour is similar to the one observed with the Eurozone assets: we denote a positive correlation with the coin token and lower than $10 \%$ positive correlation with the other (traditional) assets. Thus, it is only with the global assets of Panel $A$ that the correlation can attain a value of 0.1 with assets which are not coins. The behaviour of the low token index is exactly the same as that of the total token index, while for the mid and high token indices we find a stronger convergence towards zero for conditional correlations between tokens and traditional asset classes ${ }^{11}$. This fact confirms a certain commonality in the behaviour of the token market as a whole, as using different indices within this asset class does not change the correlation structure and portfolio diversification improvement led by tokens.

Figure 4 presents the daily dynamic conditional correlation between the coin index and the total token index on the left part, and between the (global) equity index and the token index on the right part. Their shape is totally different. The conditional correlation between coin and tokens begins at 0.4 in 2015 and attains 0.8 in 2019. It is very unstable, presenting some pseudo seasonality with a linear positive trend. On the contrary, the conditional correlation between the equities and the tokens begins at a value close to zero in 2015, and has a value close to 0.1 in 2019. Nevertheless, if on average the value oscillates around 0.1 , we have very big waves which can attain 0.4 , also with pseudo seasonality. This means there exists a positive correlation between the tokens and the coins, and a very small correlation between the tokens and the equities which can also become negative in some instances.

-Figure 6 about here-

Table 5 presents the conditional correlation across the different periods of the sample and confirms the remarks done previously. The total token index presents a high correlation with the coin index and this correlation is important since the third quantile Q75 for the period 2017-2019. The correlation is smaller if we integrate the years $2015-2016$ in the computations. On the other hand, the correlation remains very small with all the assets whatever the period except with the equities, the bonds, the real estate, gold and commodities on the periods $2017-2019$, but the value of the correlation is still very low (0.1) compared to the estimate obtained for the coin-token pairing.

-Table 5 about here-

\subsection{Diversifier, hedge or safe haven}

The conditional correlations discussed above shows evidence that tokens act as diversifier versus traditional assets and coins. In this section we employ the 'hedge or safe haven' test devised by Baur and McDermott (2010) using the estimated conditional correlations as dependent variable in a regression on dummy variables representing extreme negative movement in the time series of each of the paired assets. The results are provided in Table 6.

-Table 6 about here-

We note that we obtain highly significant and positive values for the constant term $m 0$ evolving between 0.519 (for coins) to 0.118 (for commodities) meaning that the total token index is not a

\footnotetext{
${ }^{11}$ These estimates are available upon request.
} 
good hedge against movements on all these assets, but eventually only a diversifier. The values provided for the extreme movement dummy variables confirm this fact.

Therefore, it appears that tokens are just "diversifiers" and not hedge or safe haven in welldiversified portfolios. This is according to the definitions by Baur and Lucey (2010), even if the tokens cannot be considered as a commodity and thus do not have the same status of gold (which was the asset the authors used as reference). Nevertheless, it is interesting to address the same kind of analysis done in the previous paper to tokens as new asset class in order to gain preliminary insights. As stressed earlier, these type of co-movement analyses have been previously done in the context of coins, even if cryptocurrencies and the tokens cannot be analyzed in the same way due to predictability concerns related to the former due to mining complexities.

\section{Conclusion}

In this paper we propose a first study on the evolution of the secondary market created by the rise of a new asset developed in the realm of entrepreneurial finance. Tokens are issued through an ICO and are distributed to investors in exchange for cryptocurrencies or fiat money. While only a fraction of tokens issued through an ICO are listed and actively traded, tokenized bundles of rights have by their own definition and technology the ease of trade as a key feature. Moreover, due both to their generation process and the identity of the issuer, ICO tokens are a new asset class, which lies in the realm of crypto finance but it is intrinsically different from (although related to) cryptocurrencies (also called 'coins').

We have seen the explosion of the creation of tokens between 2016 and 2018. Their numbers are huge: funding in the order of $\$ 31.5 \mathrm{Bn}$ in just 3 years of intense activity and, as a rule, without financial intermediation. In this exercise we consider 700 tokens from which we created four token indices depending on their market capitalization and their volume, and their relative dispersion across time. We provide some statistics on these four indices permitting to describe their distributional profile, and then compare these properties with classical assets traded on traditional markets. Then, we analyse the evolution of the correlation existing between these token indices and some indices based on cryptocurrencies and on different traditional asset classes, including stocks, bonds, real estate, gold and commodities, each proxied by alternative sets of indices. We observe a significant increase in the correlation between the token indices and coins but also with the other assets in the latest year of the sample. This means that the use of these tokens for hedging does not appear an interesting strategy, especially as the token market matures.

In this paper we document the sharp increase over time of the dynamic correlation between tokens and coins, reaching $80 \%$ in the latest part of our sample. Most importantly, we present evidence that tokens are not coins (i.e. fully-fledged cryptocurrencies), although they may usually be bundled as one single crypto asset category by non-attentive observers. The intrinsic value of a coin is related to its mining infrastructure and its degree of adoption as currency, while the value of a token depends, on the most part, on the success within its industry of an entrepreneurial project. Tokens have clearly a more direct link to the real economy, as they are means to fund and reap the benefits of operational ventures. We evidence that tokens do not behave neither like coins nor traditional equity instruments, and that they are effectively a new asset class. Overall, despite not being able to act as hedge or safe haven, investing in a token index will definitively make investors more diversified, even when they already have coins or Bitcoin within their portfolios.

While all the listed tokens analyzed in the paper are tradeable by retail investors, it is important to stress that proper use of an asset class within a broader portfolio can be practically achievable only for institutional investors or funds. Our results evidence that even through a complete portfolio 
allocation exercise, the token asset class can only function as a diversifier and not a hedge or safe haven vis-à-vis other assets, including cryptocurrencies.

A possible limitation of this study come from the fact that so far, tokens had on average negative returns, despite sizeable levels of underpricing at the time of listing. This might discourage investors to trade them, making them a difficult tool for portfolio allocation decisions. However, this is not the case in practice, as most tokens are actively traded despite price drops, and this is only within a time frame where the services of the companies issuing the tokens are for the most part not active. Once their platform reach operation, demand for tokens will surge as the platforms rely on them for their functioning. Furthermore, the motives for investing in ICO tokens are not purely financial (e.g. Fisch et al. 2019) and technology-driven investors and companies, make up the most important segment of token investors. What is more, any rational investors funding startups, either through VCs, crowdfunding or token purchases, has a long-term horizon in mind and knows that about $90 \%$ of startups would fail before reaching maturity. Therefore, this short to medium term negative mean return of tokens (and token indices) is not a true impediment for the trading of such asset for those who truly know what tokens are, i.e. an instrument of entrepreneurial finance which for the very first time is liquid. It is worthwhile noting that also IPO-ed stocks present significant underperformance vis-à-vis peer stocks in the medium term (e.g. Ritter, 1991), but this does not preclude the use of such stocks for their volatility contribution.

This global approach remains preliminary and needs deeper investigation to observe the evolution of this second market over longer time horizons. Such work will be pertinent to understand the evolution of the economic impact of ICOs on the real economy and financial stability. Finally, our work also stresses the importance of token indices per se, as monitoring tool of a crescent market segment. Indeed, monitoring token indices rather than individual tokens can provide a market discipline instrument for investors vis-à-vis ventures that, despite being in their infancy, have their assets listed on (crypto) exchanges. The principle guiding the construction of such token indices must be the clear understanding of what tokens are and how they systematically differ from coins. In this regard, we hope this study provided some inputs for market observers, regulators or agencies to start creating appropriate (consistent) token market indices, and ours are just a starting point.

\section{References}

Adhami, S., Giudici, G., \& Martinazzi, S. (2018). Why do businesses go crypto? An empirical analysis of initial coin offerings. Journal of Economics and Business, 100, 64-75. https://doi.org/10.1016/j.jeconbus.2018.04.001

Atzori, M. (2016) Blockchain Technology and Decentralized Governance: Is the State Still Necessary?, SSRN, 2 January 2016.

Baur, D. G., \& Lucey, B. M. (2010). Is gold a hedge or a safe haven? An analysis of stocks, bonds and gold. Financial Review, 45(2), 217-229. https://doi.org/10.1111/i.1540-6288.2010.00244.x

Baur, D. G., \& McDermott, T. K. (2010). Is gold a safe haven? International evidence. Journal of Banking \& Finance, 34(8), 1886-1898. https://doi.org/10.1016/j.jbankfin.2009.12.008

Blemus, S., \& Guégan, D. (2019). Initial Crypto-asset Offerings (ICOs), tokenization and corporate governance (No. 19004). Université Panthéon-Sorbonne (Paris 1), Centre d'Economie de la Sorbonne.

Bouri, E., Molnár, P., Azzi, G., Roubaud, D., \& Hagfors, L. I. (2017). On the hedge and safe haven 
properties of Bitcoin: Is it really more than a diversifier?. Finance Research Letters, 20, 192-198. https://doi.org/10.1016/j.frl.2016.09.025

Buterin, V. (2017) Notes on Blockchain Governance", 17 December 2017.

Cappiello, L., Engle, R. F., \& Sheppard, K. (2006). Asymmetric dynamics in the correlations of global equity and bond returns. Journal of Financial econometrics, 4(4), 537-572. https://doi.org/10.1093/ijfinec/nbl005

Cho, J. H., \& Parhizgari, A. M. (2008). East Asian financial contagion under DCC-GARCH. International Journal of Banking and Finance, 6(1), 17-30.

Engle, R. (2002). Dynamic conditional correlation: A simple class of multivariate generalized autoregressive conditional heteroskedasticity models. Journal of Business \& Economic Statistics, 20(3), 339-350. https://doi.org/10.1198/073500102288618487

Fenu, G., Marchesi, L., Marchesi, M., \& Tonelli, R. (2018, March). The ICO phenomenon and its relationships with ethereum smart contract environment. In 2018 International Workshop on Blockchain Oriented Software Engineering (IWBOSE) (pp. 26-32). IEEE.

Fisch, C. (2019). Initial coin offerings (ICOs) to finance new ventures. Journal of Business Venturing, 34(1), 1-22. https://doi.org/10.1016/i.jbusvent.2018.09.007

Fisch, C., Masiak, C., Vismara, S., \& Block, J. (2019). Motives and profiles of ICO investors. Journal of Business Research. https://doi.org/10.1016/j.jbusres.2019.07.036

Folks, J. L., \& Chhikara, R. S. (1978). The inverse Gaussian distribution and its statistical application-a review. Journal of the Royal Statistical Society: Series B (Methodological), 40(3), 263-275. https://doi.org/10.1111/i.2517-6161.1978.tb01039.x

Giudici, G., \& Adhami, S. (2019). The impact of governance signals on ICO fundraising success. Journal of Industrial and Business Economics, 46(2), 283-312. https://doi.org/10.1007/s40812-019$\underline{00118-w}$

Howell, S. T., Niessner, M., \& Yermack, D. (2018). Initial coin offerings: Financing growth with cryptocurrency token sales (No. w24774). National Bureau of Economic Research.

Huang, W., Meoli, M., \& Vismara, S. (2018). The geography of initial coin offerings. Small Business Economics, 1-26. https://doi.org/10.1007/s11187-019-00135-y

Lee, J., Li, T., \& Shin, D. (2018). The Wisdom of Crowds and Information Cascades in FinTech: Evidence from Initial Coin Offerings. https://ssrn.com/abstract=3226051

Li, J., \& Mann, W. (2018). Initial coin offering and platform building. https://dx.doi.org/10.2139/ssrn.3088726

Ratner, M., \& Chiu, C. C. J. (2013). Hedging stock sector risk with credit default swaps. International Review of Financial Analysis, 30, 18-25. https://doi.org/10.1016/j.irfa.2013.05.001

Ritter, J. R. (1991). The long-run performance of initial public offerings. The journal of finance, 46(1), 3-27. https://doi.org/10.1111/j.1540-6261.1991.tb03743.x

Wong, W. S., Saerback, D., \& Delgado Silva, D. (2018). Cryptocurrency: A New Investment Opportunity? An Investigation of the Hedging Capability of Cryptocurrencies and Their Influence on Stock, Bond and Gold Portfolios. https://dx.doi.org/10.2139/ssrn.3125737

Shleifer, A., \& Vishny, R. W. (1997). A survey of corporate governance. The journal of finance, 52(2), 737-783. https://doi.org/10.1111/j.1540-6261.1997.tb04820.x 
Shuster, J. (1968). On the inverse Gaussian distribution function. Journal of the American Statistical Association, 63(324), 1514-1516.

Trimborn, S., \& Härdle, W. K. (2018). CRIX an Index for cryptocurrencies. Journal of Empirical Finance, 49, 107-122. https://doi.org/10.1016/i.jempfin.2018.08.004

Urquhart, A., \& Zhang, H. (2019). Is Bitcoin a hedge or safe haven for currencies? An intraday analysis. International Review of Financial Analysis. https://doi.org/10.1016/j.irfa.2019.02.009

Yadav, M. (2017). Exploring signals for investing in an Initial Coin Offering (ICO). Available at SSRN 3037106. 


\section{Figures}

Figure 1: The ICO Market Vs. Bitcoin price and movements. The daily volatility of the BTC return series is computed quarterly, on a rolling basis. The scale of the daily BTC volatility has been adjusted for presentation purposes. Data sources: CoinMarketCap.com and ICOData.io.

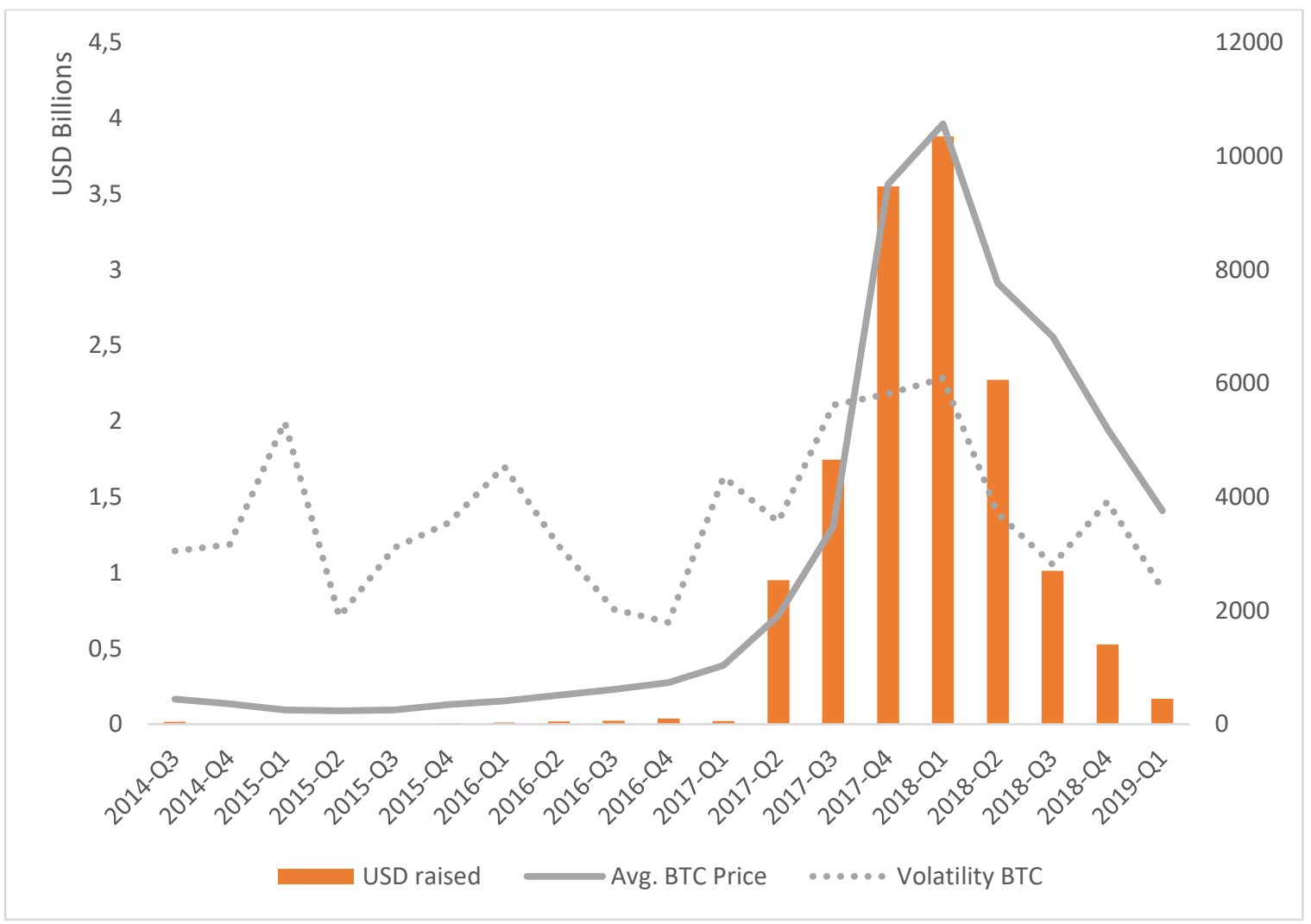

Figure 2: The ICO market timeline: amounts raised, completed ICO fundraisings and ICO token listings on secondary markets. Figures and values per quarter, not cumulated. Data sources: ICOData.io and proprietary dataset. 


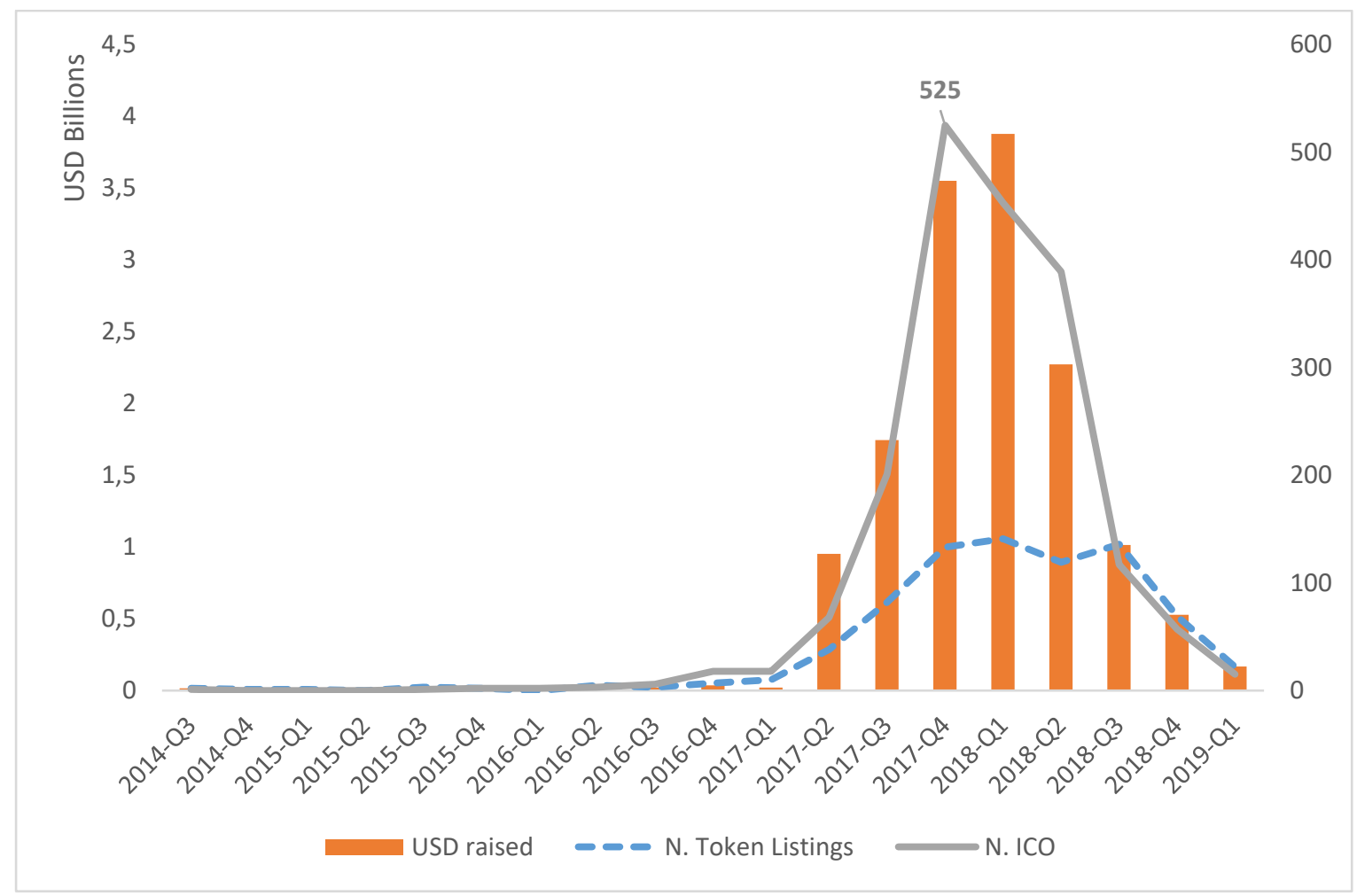

Figure 3: Price dynamics of the token indices.

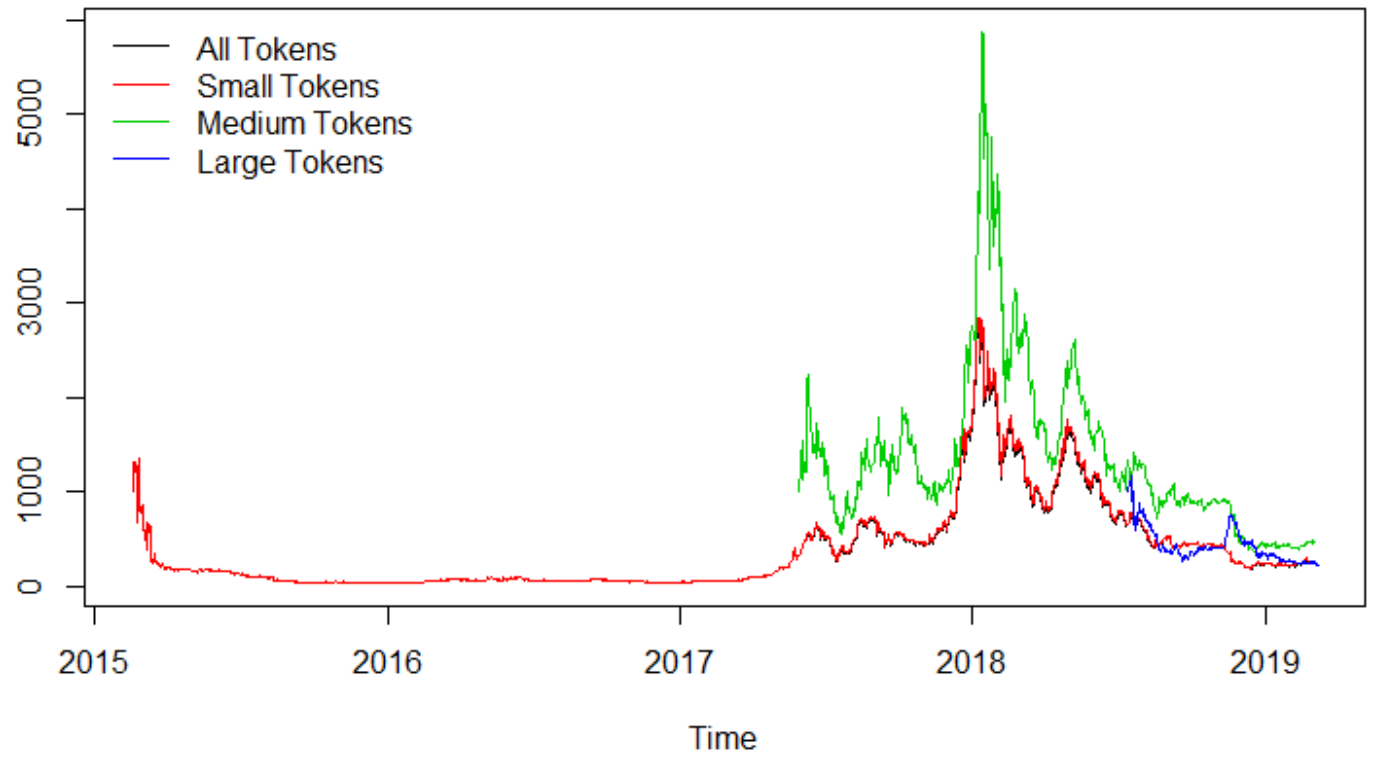

Figure 4: Price dynamics of major crypto assets. 


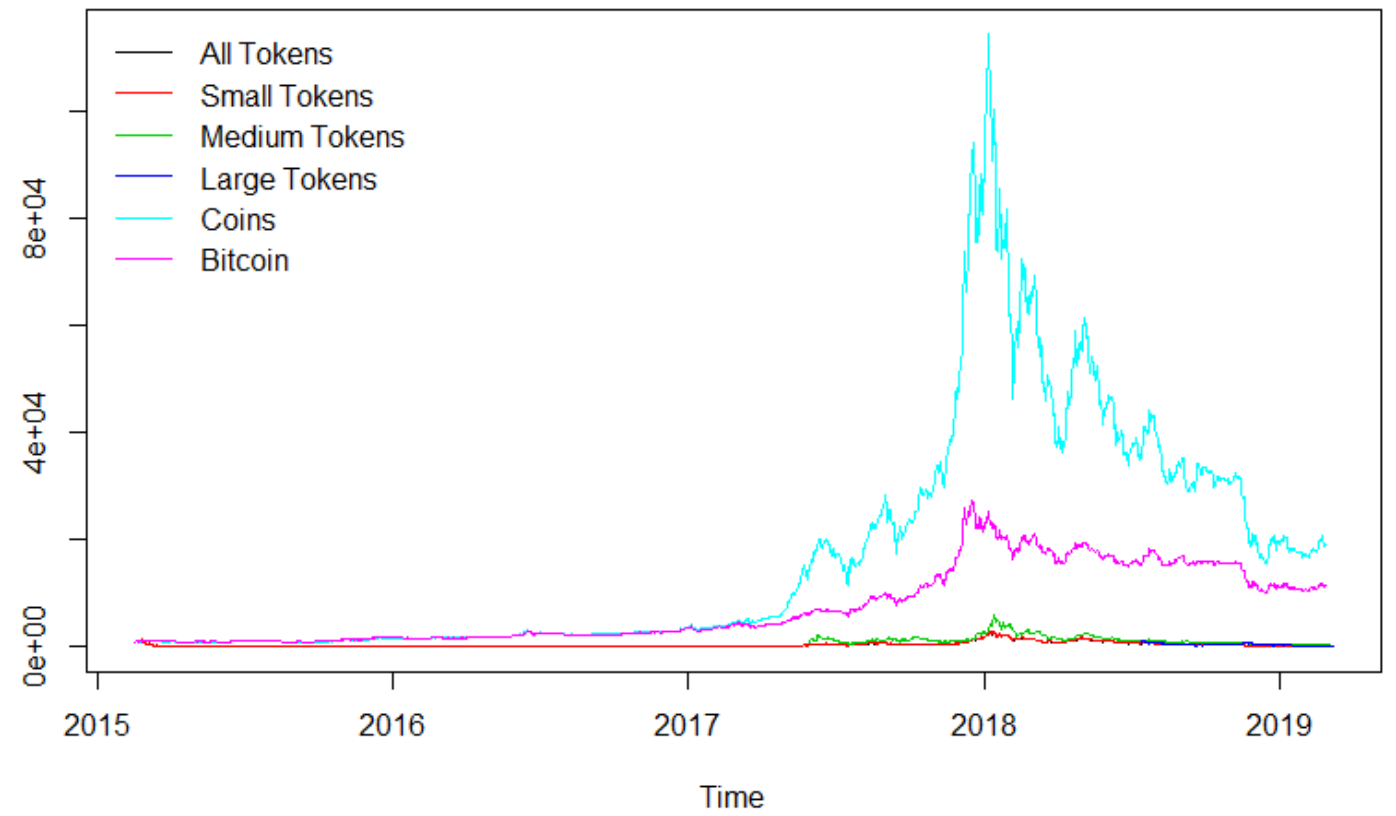

Figure 5: Daily returns of the token index, the cryptocurrency index (i.e. "coins") and the Bitcoin.

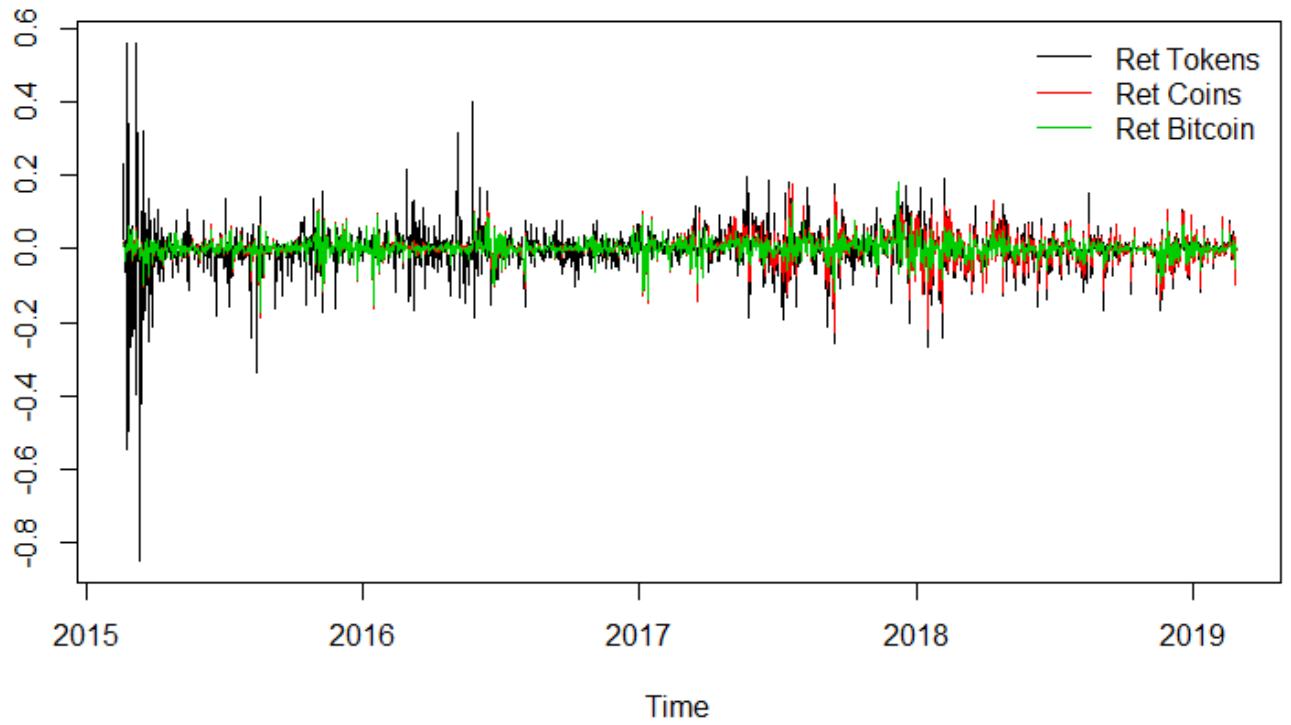

Figure 6: Daily dynamic conditional correlation estimates for the pairs coins-tokens and equitiestokens. 
DCC: Coins vs. Tokens

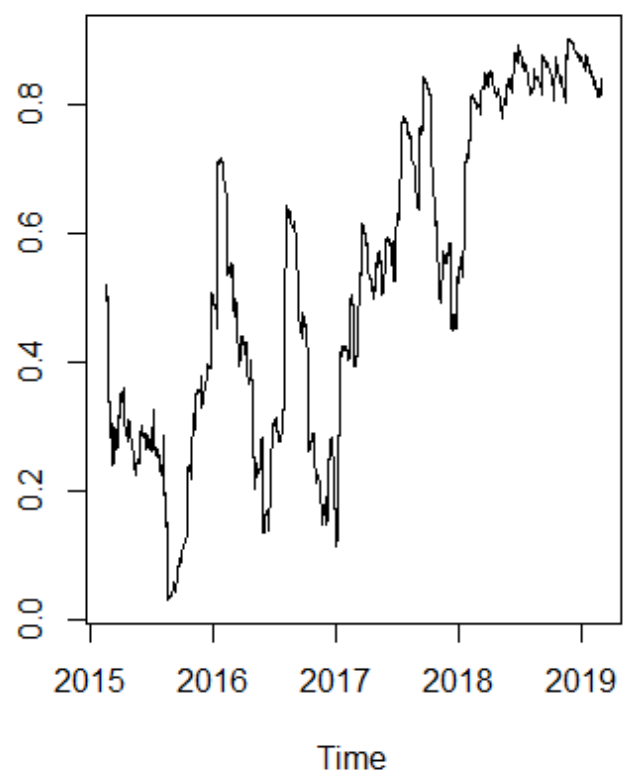

DCC: Equities vs. Tokens

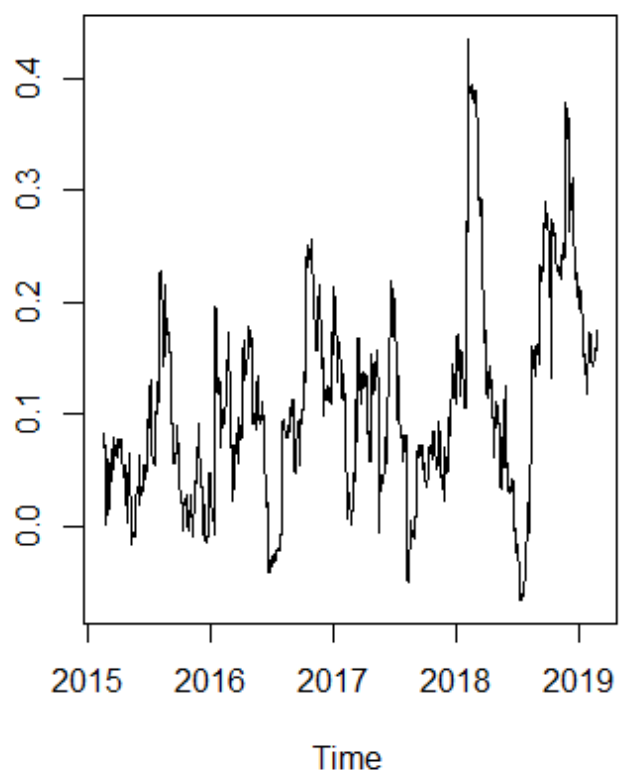

\section{Tables}

Table 1: Description of the sample of tokens populating the indices presented in the paper.

\begin{tabular}{|c|c|c|c|c|}
\hline & Total Index & Low Index & Mid Index & High Index \\
\hline \multicolumn{5}{|c|}{ Regional/Country Distribution } \\
\hline Europe & $33.0 \%$ & $32.2 \%$ & $38.9 \%$ & $34.6 \%$ \\
\hline North America & $19.9 \%$ & $23.0 \%$ & $17.4 \%$ & $7.7 \%$ \\
\hline Singapore & $13.9 \%$ & $14.9 \%$ & $11.8 \%$ & $3.8 \%$ \\
\hline Other Microstates & $8.8 \%$ & $6.6 \%$ & $9.7 \%$ & $15.4 \%$ \\
\hline Russia & $5.5 \%$ & $5.6 \%$ & $5.6 \%$ & $7.7 \%$ \\
\hline China & $4.1 \%$ & $3.7 \%$ & $4.2 \%$ & $7.7 \%$ \\
\hline Other & $14.8 \%$ & $13.9 \%$ & $12.5 \%$ & $23.1 \%$ \\
\hline \multicolumn{5}{|l|}{ Amount Raised (USD) } \\
\hline average & $17,505,385$ & $28,311,705$ & $15,395,508$ & $8,808,943$ \\
\hline total & $17,321,770,377$ & $14,750,398,322$ & $2,324,721,644$ & $246,650,411$ \\
\hline \multicolumn{5}{|c|}{ Token and ICO Characteristics } \\
\hline avg. 'experts' rating & 3.2 & 3.1 & 3.4 & 3.7 \\
\hline \%tokens distributed (avg.) & $51.3 \%$ & $51.0 \%$ & $54.3 \%$ & $41.2 \%$ \\
\hline \%Ethereum (avg.) & $88.9 \%$ & $90.0 \%$ & $89.1 \%$ & $81.5 \%$ \\
\hline payment token & $3.5 \%$ & $4.2 \%$ & $2.7 \%$ & $3.7 \%$ \\
\hline security token & $0.8 \%$ & $0.6 \%$ & $0.7 \%$ & $0.0 \%$ \\
\hline utility token & $95.7 \%$ & $95.2 \%$ & $96.6 \%$ & $96.3 \%$ \\
\hline Total & 700 & 521 & 151 & 28 \\
\hline
\end{tabular}



Table 2: Descriptive statistics of the main (daily return) time series used in the paper. The sample starts on February $18^{\text {th }} 2015$ and ends in February $27^{\text {th }} 2019$ for all the series with an exception for the Mid-Token-Index and the High-Token-Index, which start respectively on May $25^{\text {th }} 2017$ and July $5^{\text {th }}$ 2018. 'Rho' represents the first order autocorrelation coefficient. The last two columns present, respectively, the p-values of the Augmented Dickey-Fuller test and the 12-lag Ljung-Box test.

\begin{tabular}{|c|c|c|c|c|c|c|c|c|c|}
\hline Series & $\begin{array}{r}\text { Mean } \\
\%\end{array}$ & SD \% & Skew & Kurt & Min \% & Max \% & Rho & ADF Test & LB Test \\
\hline \multicolumn{10}{|l|}{ 1.Tokens } \\
\hline -total index & -0.091 & 7.110 & -1.246 & 25.266 & -84.842 & 56.020 & -0.146 & 0.010 & 0.000 \\
\hline -low index & -0.089 & 7.113 & -1.240 & 25.231 & -84.842 & 56.020 & -0.146 & 0.010 & 0.000 \\
\hline -mid index & -0.117 & 7.472 & 0.331 & 4.448 & -27.928 & 52.830 & -0.020 & 0.010 & 0.446 \\
\hline -high index & -0.620 & 6.932 & -0.428 & 1.741 & -25.579 & 20.946 & -0.147 & 0.010 & 0.236 \\
\hline 2.Coins & 0.201 & 3.778 & -0.586 & 4.791 & -22.777 & 17.764 & -0.015 & 0.010 & 0.015 \\
\hline \multicolumn{10}{|l|}{ 3.Equities } \\
\hline -Global & 0.012 & 0.613 & -0.946 & 7.802 & -5.135 & 2.757 & 0.077 & 0.010 & 0.000 \\
\hline -Eurozone & -0.002 & 0.838 & -0.634 & 8.046 & -7.081 & 4.103 & -0.041 & 0.010 & 0.004 \\
\hline -US & 0.019 & 0.715 & -0.540 & 7.305 & -4.184 & 4.840 & -0.029 & 0.010 & 0.026 \\
\hline \multicolumn{10}{|l|}{ 4.Bonds } \\
\hline -Global & 0.003 & 0.422 & -0.661 & 9.004 & -3.920 & 2.687 & -0.042 & 0.010 & 0.222 \\
\hline -Eurozone & 0.004 & 0.098 & -0.539 & 4.783 & -0.723 & 0.491 & 0.073 & 0.010 & 0.094 \\
\hline -US & 0.013 & 0.077 & 0.503 & 4.885 & -0.410 & 0.527 & 0.024 & 0.010 & 0.855 \\
\hline 5.Real Estate & -0.001 & 0.625 & -0.726 & 4.608 & -4.374 & 2.444 & 0.087 & 0.010 & 0.018 \\
\hline 6.Gold & 0.004 & 1.833 & -0.196 & 4.777 & -11.893 & 9.100 & -0.077 & 0.010 & 0.000 \\
\hline 7.Commodities & 0.001 & 1.047 & -0.053 & 3.228 & -5.034 & 5.258 & -0.060 & 0.010 & 0.155 \\
\hline
\end{tabular}


Table 3: The performance criteria of the different specifications of the DCC- and ADCC-GARCH models under multivariate Student's T distribution. The ARMA $(p, q)$ models for the underlying series range from $[p=0, q=0]$ to $[p=2, q=2]$ according to a previous round of optimization. The optimal model is in bold.

\begin{tabular}{|c|c|c|c|}
\hline Model. & AIC & $\mathrm{BIC}$ & HQC \\
\hline $\begin{array}{l}\operatorname{DCC-ARMA}(1,1)- \\
\operatorname{GARCH}(1,1)\end{array}$ & -44.173 & -43.910 & -44.075 \\
\hline $\begin{array}{l}\operatorname{DCC-ARMA}(1,1)- \\
\text { GJRGARCH }(1,1)\end{array}$ & -44.219 & -43.931 & -44.112 \\
\hline $\begin{array}{l}\operatorname{DCC}-\operatorname{ARMA}(1,1)- \\
\operatorname{EGARCH}(1,1)\end{array}$ & -42.880 & -42.592 & -42.772 \\
\hline $\begin{array}{l}\operatorname{ADCC}-\operatorname{ARMA}(1,1)- \\
\operatorname{GARCH}(1,1)\end{array}$ & -44.196 & -43.929 & -44.097 \\
\hline $\begin{array}{l}\text { ADCC-ARMA(1,1)- } \\
\operatorname{GJRGARCH}(1,1)\end{array}$ & -44.245 & -43.953 & -44.136 \\
\hline $\begin{array}{l}\operatorname{ADCC}-\operatorname{ARMA}(1,1)- \\
\operatorname{EGARCH}(1,1)\end{array}$ & -42.942 & -42.651 & -42.833 \\
\hline $\begin{array}{l}\text { DCC-ARMA }(p, q)- \\
\operatorname{GARCH}(1,1)\end{array}$ & -44.166 & -43.903 & -44.068 \\
\hline $\begin{array}{l}\operatorname{DCC-ARMA}(p, q)- \\
\operatorname{GJRGARCH}(1,1)\end{array}$ & -44.208 & -43.920 & -44.100 \\
\hline $\begin{array}{l}\operatorname{DCC}-\operatorname{ARMA}(p, q)- \\
\operatorname{EGARCH}(1,1)\end{array}$ & -42.934 & -42.646 & -42.827 \\
\hline $\begin{array}{l}\text { ADCC-ARMA(p,q)- } \\
\operatorname{GARCH}(1,1)\end{array}$ & -44.189 & -43.922 & -44.089 \\
\hline $\begin{array}{l}\text { ADCC-ARMA(p,q)- } \\
\text { GJRGARCH }(1,1)\end{array}$ & -44.233 & -43.942 & -44.125 \\
\hline $\begin{array}{l}\operatorname{ADCC}-\operatorname{ARMA}(p, q)- \\
\operatorname{EGARCH}(1,1)\end{array}$ & -42.998 & -42.707 & -42.890 \\
\hline
\end{tabular}


Table 4: Descriptive statistics of the daily dynamic conditional correlations (DCC) between each asset and the (total) token index estimated from the optimal MGARCH model. The sample starts on February $18^{\text {th }} 2015$ and ends in February $27^{\text {th }} 2019$ for all the series.

\begin{tabular}{|c|c|c|c|c|c|c|c|}
\hline Series & Mean & SD & Min & Q25 & Median & Q75 & Max \\
\hline \multicolumn{8}{|c|}{ Panel A: Global Assets } \\
\hline Coins & 0.532 & 0.250 & 0.030 & 0.293 & 0.533 & 0.807 & 0.902 \\
\hline Equities & 0.108 & 0.091 & -0.067 & 0.045 & 0.093 & 0.154 & 0.436 \\
\hline Bonds & 0.135 & 0.079 & -0.017 & 0.073 & 0.130 & 0.174 & 0.352 \\
\hline Real Estate & 0.067 & 0.093 & -0.110 & -0.001 & 0.054 & 0.120 & 0.395 \\
\hline Gold & 0.103 & 0.069 & -0.126 & 0.067 & 0.099 & 0.141 & 0.330 \\
\hline Commodities & 0.117 & 0.063 & -0.048 & 0.075 & 0.118 & 0.158 & 0.296 \\
\hline \multicolumn{8}{|c|}{ Panel B: Eurozone Assets } \\
\hline Coins & 0.513 & 0.253 & 0.024 & 0.275 & 0.518 & 0.794 & 0.891 \\
\hline Equities & 0.073 & 0.088 & -0.118 & 0.010 & 0.066 & 0.119 & 0.364 \\
\hline Bonds & 0.082 & 0.080 & -0.121 & 0.036 & 0.077 & 0.130 & 0.326 \\
\hline Real Estate & 0.048 & 0.091 & -0.137 & -0.019 & 0.035 & 0.096 & 0.327 \\
\hline Gold & 0.071 & 0.068 & -0.149 & 0.036 & 0.065 & 0.114 & 0.273 \\
\hline Commodities & 0.085 & 0.056 & -0.080 & 0.048 & 0.089 & 0.125 & 0.229 \\
\hline \multicolumn{8}{|c|}{ Panel C: US Assets } \\
\hline Coins & 0.510 & 0.248 & 0.062 & 0.289 & 0.477 & 0.782 & 0.891 \\
\hline Equities & 0.063 & 0.074 & -0.053 & 0.015 & 0.041 & 0.104 & 0.334 \\
\hline Bonds & 0.098 & 0.066 & -0.045 & 0.053 & 0.091 & 0.133 & 0.287 \\
\hline Real Estate & 0.059 & 0.078 & -0.079 & 0.005 & 0.051 & 0.099 & 0.279 \\
\hline Gold & 0.080 & 0.058 & -0.055 & 0.044 & 0.070 & 0.119 & 0.256 \\
\hline Commodities & 0.093 & 0.044 & -0.035 & 0.065 & 0.097 & 0.121 & 0.208 \\
\hline
\end{tabular}


Table 5: Descriptive statistics of the daily dynamic conditional correlations (DCC) between each asset and the (total) token index estimated from the optimal MGARCH model. The sample starts on February $18^{\text {th }} 2015$ and ends in February $27^{\text {th }} 2019$ for all the series.

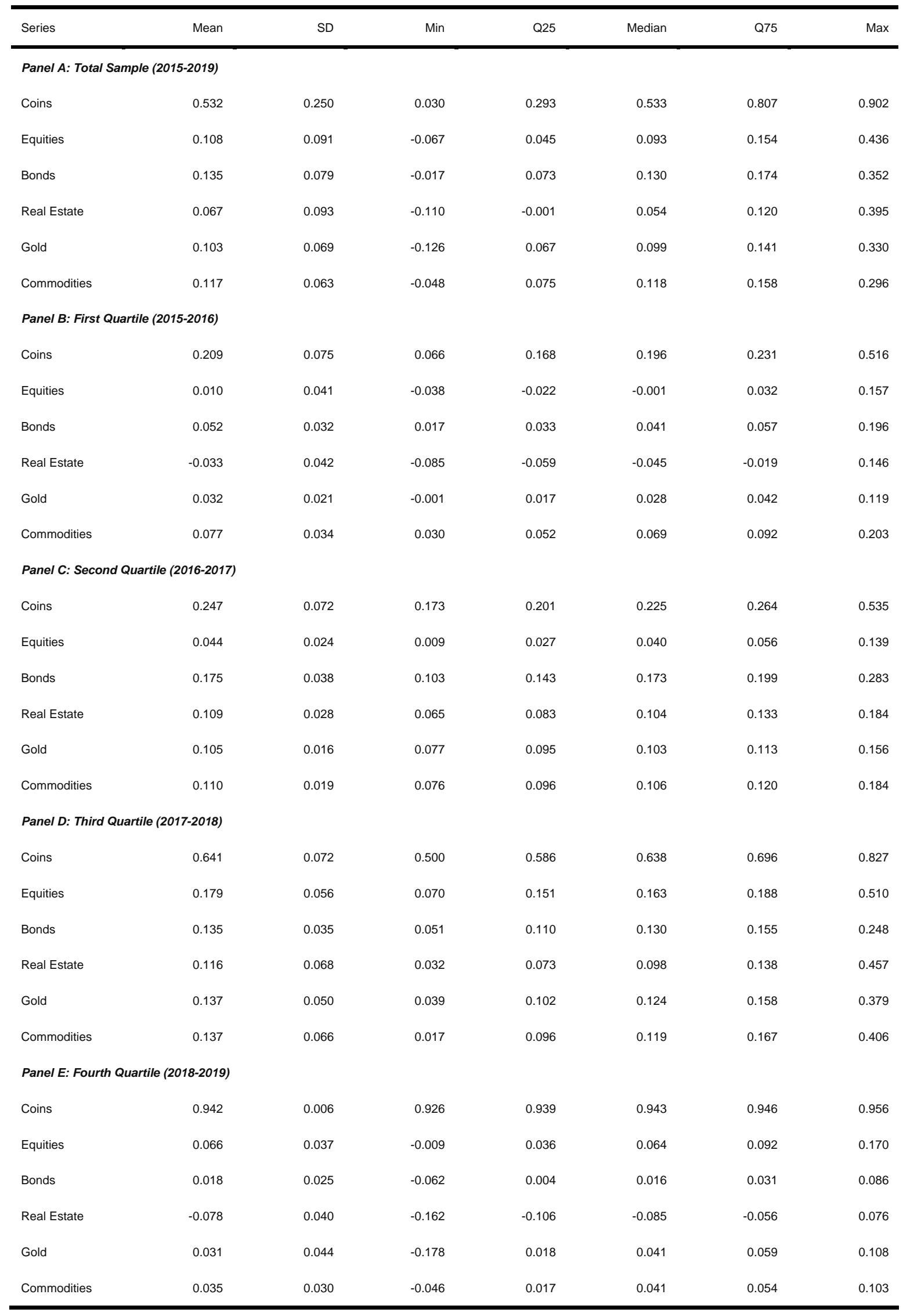


Table 6: Estimation results on hedge and safe haven properties of tokens for daily returns. Tokenized assets are represented by the Total Token Index. Negative coefficients for $m 0$ would indicate that tokens act as hedge against the paired asset. Zero (negative) coefficients in extreme market conditions (i.e. $m 1, m 2$ or $m 3$ representing the lowest $10^{\text {th }}, 5^{\text {th }}$ and $1^{\text {st }}$ percentiles) would indicate that tokens are a weak (strong) safe haven.

\begin{tabular}{lccccccc}
\hline & \multicolumn{7}{c}{ Dependent variable: } \\
\cline { 2 - 8 } & & \multicolumn{7}{c}{ DCC paired with Tokens } \\
& $(1)$ & $(2)$ & $(3)$ & $(4)$ & $(5)$ & $(6)$ \\
\hline $\mathrm{m} 1$ & $0.142^{* * * *}$ & 0.002 & -0.010 & $0.023^{* *}$ & -0.011 & -0.006 \\
& $(0.030)$ & $(0.011)$ & $(0.010)$ & $(0.011)$ & $(0.008)$ & $(0.008)$ \\
$\mathrm{m} 2$ & -0.017 & 0.016 & 0.008 & $-0.028^{*}$ & -0.013 & 0.002 \\
& $(0.043)$ & $(0.016)$ & $(0.014)$ & $(0.016)$ & $(0.012)$ & $(0.011)$ \\
$\mathrm{m} 3$ & & & & & & \\
& -0.089 & 0.027 & -0.011 & 0.033 & 0.015 & -0.001 \\
$\mathrm{~m} 0$ & $(0.071)$ & $(0.026)$ & $(0.023)$ & $(0.027)$ & $(0.020)$ & $(0.018)$ \\
& $0.519^{* * * *}$ & $0.107^{* * * *}$ & $0.136^{* * *}$ & $0.065^{* * *}$ & $0.105^{* * *}$ & $0.118^{* * *}$ \\
& $(0.007)$ & $(0.002)$ & $(0.002)$ & $(0.003)$ & $(0.002)$ & $(0.002)$ \\
& & & & & & \\
\hline Observations & 1,470 & 1,470 & 1,470 & 1,470 & 1,470 & 1,470 \\
Residual Std. Error (df = 1466) & 0.247 & 0.091 & 0.079 & 0.093 & 0.069 & 0.063 \\
F Statistic (df = 3; 1466) & $11.919^{* * * *}$ & 1.982 & 0.556 & 1.909 & $2.914^{* *}$ & 0.346 \\
& & & & & & \\
\hline Note: & & & & & $\mathrm{p}^{* * *} \mathrm{p}^{* * *} \mathrm{p}<0.01$
\end{tabular}

